Journal of the Optical Society of Korea, Vol. 10, No. 2, June 2006, pp. 76-80

\title{
Stereoscopic Floating Image System Using Stereoscopic Display and Two Lenses
}

\author{
Ganbat Baasantseren*, Duc Dung Do, Ki-Cheol Kwon, and Nam Kim \\ Dept. of Computer \& Communication Eng., Chungbuk Nat'l Univ., KOREA
}

(Received April 21, 2006 : revised May 25, 2006)

\begin{abstract}
The proposed technique uses a combination of two systems, viz. a three dimensional stereoscopic system and a two-lens system. This novel combined system successfully produces a stereoscopic floating image in mid air near the observer. The two-lens system produces a floating image from the stereoscopic image originating from the stereoscopic system, and the two lenses eliminate the defects of the floating lens and concave mirror. The experimental results show that the two lenses eliminate the defects of the lens and the concave mirror, so that the proposed system successfully produces a touchable stereoscopic floating image.

OCTS codes : $111.6880,170.0110,330.1800$
\end{abstract}

\section{INTRODUCTION}

Floating-Image Displays have many applications including advertising and promotion, collaborative decision making and simulation, because these applications need a touchable 3D image like the real object. Today, there are many kinds of $3 \mathrm{D}$ displays, but the $3 \mathrm{D}$ images that they produce are not touchable. Therefore, many researchers are studying how to produce a floating image from a 3D image. In floating image displays, a large concave mirror or large lens is used to produce the floating image. However, the lens and concave mirror produce a defective image, because the magnifications of these two elements are not constant and the image distance is not linearly related to the object distance.

In this paper, we present a stereoscopic floating image system using a stereo display and two lenses. The two-lens system can display floating images a large convex lens or a concave mirror. The proposed floating image display provides an impressive feel of depth, and the produced image appears to be located in free space near the observer [1]. The stereoscopic display adopts the binocular disparity [7], because it provides good feeling of depth.

This paper is organized as follows. In Section II, we present the polarization-multiplexed display. Section III presents the defects of the floating lens and discusses the two-lens system. Section IV presents the experimental results obtained with the stereoscopic floating display system. Finally, we make our conclusions in Section V.

\section{STEREOSCOPIC DISPLAY TECHNIQUES}

To perceive a scene in stereo $3 \mathrm{D}$, humans use two eyes in parallel on a plane perpendicular to that of the image (binocular vision). As the eyes are set apart (by $6.4 \mathrm{~cm}$ on average), they view the environment from slightly different perspectives. The different images of a scene produced by the left and right eyes are called a stereo pair. Small differences in the location of corresponding points in the stereo pair images [11] give accurate information about the depth of objects in relation to each other. This difference is termed binocular disparity and is used by the brain to produce the effect of depth in the scene. Stereopsis is therefore the process of perceiving depth in an environment using images produced by binocular vision.

There are four basic techniques used in stereoscopic displays, viz. Color (anaglyph) [4,5,10], Polarization multiplexed $[4,5,10]$, Time multiplexed $[9,10]$ and Location multiplexed (HMD) [8]. Among these four techniques, one advantage of the polarization-multiplexed technique is that the observer can view the stereo image in full color and full resolution and does not need expensive active glasses. Also, polarization multiplexed displays are becoming fairly inexpensive and can have multiple viewers (each with their own headset) [12].

The polarization multiplexed display simultaneously presents the left image and the right image to the viewer. This display structure is illustrated in figure 1. In the two-display set-up, the left monitor creates the left image that is polarized at $45^{\circ}$, and the right image 


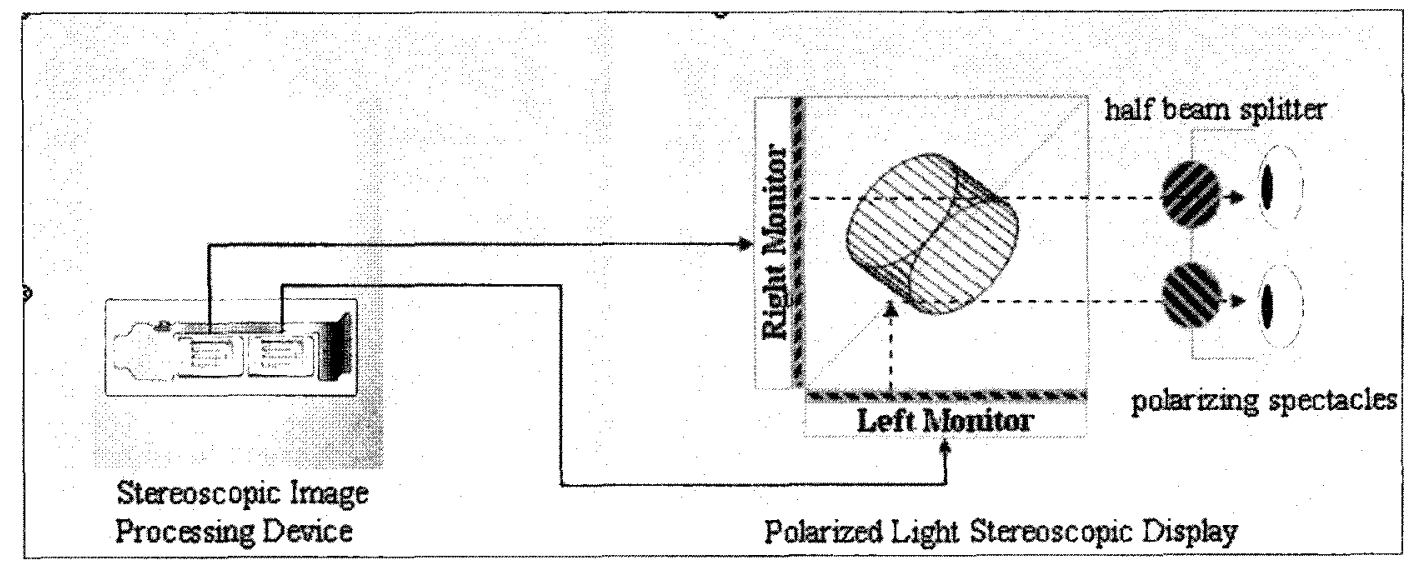

FIG. 1. Polarization multiplexed stereoscopic display structure.

monitor creates the right image that is polarized at $135^{\circ}$. A beam splitter (semi reflector) is used to channel the left and right images to the observer. When the stereo pair images from the two monitors are viewed through crossed-polarizing glasses, the left eye only sees the left image and the right eye only sees the right image. This allows the visual system to merge the two images, resulting in the perception of depth, or stereopsis.

\section{LENS DEFECTS AND TWO LENSES}

The convex lens and concave mirror produce defective images of objects, because their magnifications are not constant, and the image distance is not linearly related to the object distance. Herein, we discuss only the defects of the convex lens, because the problems posed by the concave mirror and convex lens are very similar. There are two kinds of defects associated with the convex lens: the height defect and distance defect.

For example in Fig. 2 (a), although the heights of the two objects are different, the heights of their respective images are equal $\left(h_{1} \neq h_{2}\right.$ but $\left.h_{1}^{*}=h_{2}^{*}\right)$, while in Fig. 2 (b), the heights of the two objects are equal, but the heights of their images are not equal $\left(h_{1}=h_{2}\right.$ but $\mathrm{h}_{1}{ }_{1} \neq \mathrm{h}_{2}^{*}$ )

In the case of the distance defect, the distance between the two objects differs from the distance between their images. For example in Fig. 2 (c), a and $a_{2}$ are equal, but $a_{1}^{*}$ and $a_{2}^{*}$ are not equal, where $a_{1}, a_{2}$ are the distances between $\mathrm{O}_{1}, \mathrm{O}_{2}$ and $\mathrm{O}_{2}, \mathrm{O}_{3}$, respectively, and $\mathrm{a}_{1}^{*}, \mathrm{a}_{2}{ }_{2}$ are the distances between $\mathrm{i}_{1}, \mathrm{i}_{2}$ and $\mathrm{i}_{2}, \mathrm{i}_{3}$, respectively.

The floating image system has two kinds of magnification. The first is the height magnification, which is defined as

$$
M_{h}=\frac{\text { imageheight }}{\text { objectheight }} .
$$

The second is the distance magnification, which is

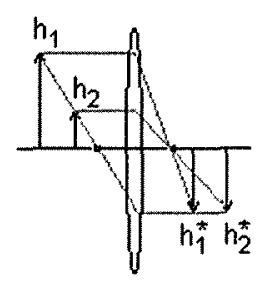

(a)

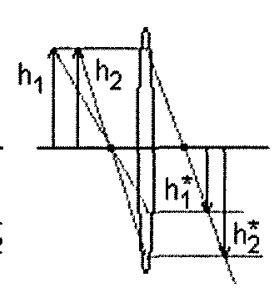

(b)

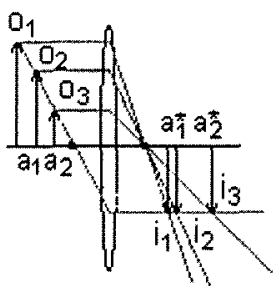

(c)
FIG. 2. Lens defects. (a) The heights of the two objects are different, but the heights of their images are equal. (b) The heights of the two objects are equal, but the heights of their images are different. (c) The distances between the objects are equal, but the distances between the images are not equal.

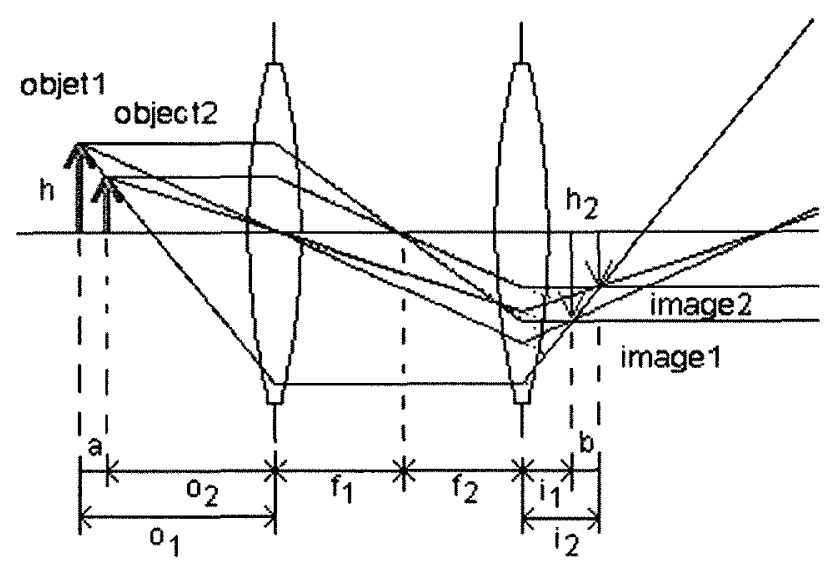

FIG. 3. Formation of two images using two lenses.

defined as

$$
M_{d}=\frac{\text { imagedis } \tan c e}{\text { objectdis } \tan c e} .
$$

The use of two lenses eliminates these lens defects, as shown in Fig. 3. When the distance between the two lenses is equal to the sum of their focal lengths, the 
two lenses eliminate all of the lens defects $\left(d=f_{1}+f_{2}\right.$ : where $\mathrm{d}$ is the distance between the two lenses, and $f_{1}$ and $f_{2}$ are the focal lengths of the first and second lenses, respectively).

According to Eq. 1, the height magnification in Fig. 4 is described by Eq. 3

$$
\frac{h_{2}}{h}=\frac{f_{2}}{f_{1}} \Rightarrow M_{h}=\frac{f_{2}}{f_{1}},
$$

where $h$ is the height of object $1, h_{2}$ is the height of image $1, f_{1}$ is the focal length of lens 1 and $f_{2}$ is the focal length of lens 2 .

The relation between the image distance and object distance is given by

$$
i=\frac{f_{1} f_{2}\left(f_{2}+f_{1}\right)-f_{2}^{2} o}{f_{1}^{2}},
$$

where $o$ is the object distance, and $i$ is the image distance.

According to Eqs. 2 and 4, we can determine the distance magnification as follows :

$$
M_{d}=\frac{i_{1}-i_{2}}{o_{1}-o_{2}}=-\frac{f_{2}^{2}}{f_{1}^{2}},
$$

where $o_{1}$ is the distance of object 1 from lens $1, i_{1}$ is the distance of image 1 from lens $2, \mathrm{O}_{2}$ is the distance of object 2 from lens 1 , and $i_{2}$ is the distance of image 2 from lens 2. The distance magnification $\left(\mathrm{M}_{\mathrm{d}}=-\frac{f_{2}^{2}}{f_{1}^{2}}\right)$ and the height magnification $\left(\mathrm{M}_{\mathrm{h}}=\frac{f_{2}}{f_{1}}\right)$ are equal when the focal lengths of the two lenses are equal $\left(f_{1}=f_{2}\right)$. Thus, we can rewrite Eq. 4 as

$$
i=2 f-o \text {. }
$$

From Eq. 6, we can see that the object position range is $0<0<2 f$, the image distance range is $2 \mathrm{f}>\mathrm{i}>0$, and the image maximum distance is $2 \mathrm{f}$.

We can compare the two lenses system with the

TABLE 1. Comparison of two lenses system with floating lens system.

\begin{tabular}{l|c|c}
\hline \hline \multicolumn{1}{c|}{ Parameters } & Two lenses & Floating lens \\
\hline $\begin{array}{l}\text { Relationship between } \\
\text { image and object distance }\end{array}$ & $i=2 f-o$ & $i=\frac{o f}{o-f}$ \\
\hline Image range & $0<\mathrm{i}<2 \mathrm{f}$ & $0<\mathrm{i}<\mathrm{f}$ \\
\hline Image position & controllable & fixed \\
\hline Height magnification & constant & not constant \\
\hline Distance magnification & constant & not constant \\
\hline Magnifications defend on & $\mathrm{f}$ & $\mathrm{f}, \mathrm{o}, \mathrm{i}$ \\
\hline
\end{tabular}

floating lens system, as shown in table 1.

\section{EXPERIMENT}

We conducted the experiment in two steps. In the first step, we checked the distance and height magnification of the two-lens system and the reducing defects of the floating lens system. In the second step, we merged the two-lens system with the stereoscopic display.

In the experiment, we used two Fresnel lenses and a camera, as shown in Fig. 4 (a). The focal lengths of the two Fresnel lenses are both $230 \mathrm{~mm}$ and the distance between them is equal to the sum of their focal lengths. The two lenses produce an image of an object that is rotated by $180^{\circ}$. According to Eq. 6 , the object range is $0<0<460 \mathrm{~mm}$, so the image distance range is $460 \mathrm{~mm}>\mathrm{i}>0$, and the maximum image distance is $460 \mathrm{~mm}$.

The original object and the compared object are two identical papers. The width and height of the small quadrates on the papers are $5 \mathrm{~mm}$. The right paper is the original object, and the left paper is the compared object that is on the line. The height magnification of the two lenses is equal to one, so the image size is the same as the object size. The experimental result is shown in Fig. 5.

The sizes of the left and right papers appear to be the same, so we can say that the height magnification of the two-lens system is equal to one.

We took three pictures after changing the object

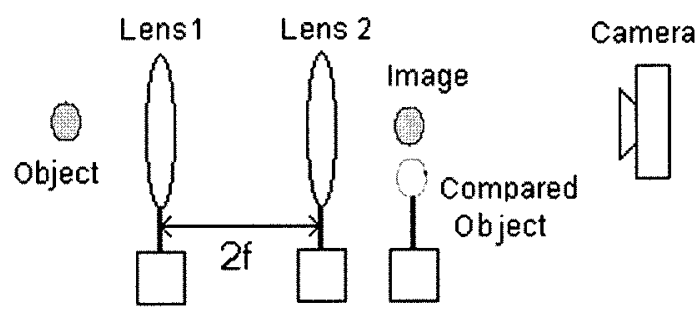

FIG. 4. Experimental set-up.

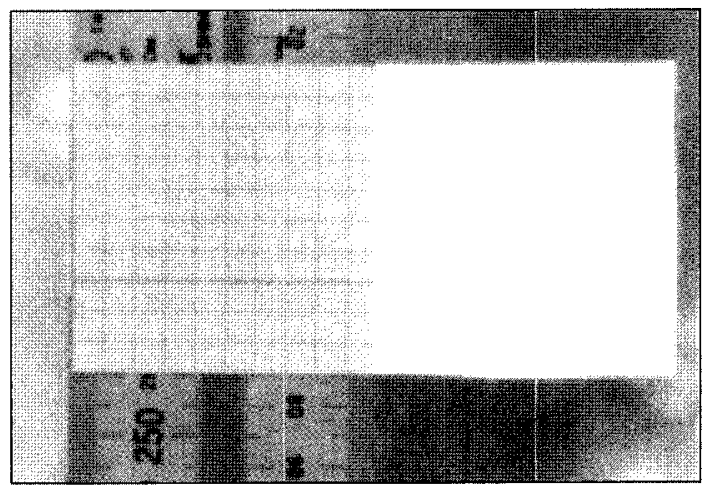

FIG. 5. Height magnification. 
distance, as shown in Fig. 6. We always focused on the compared object and shifted the position of the object. In Fig. 6 (a), the object distance is $200 \mathrm{~mm}$ when the compared object and the floating image are in the same plane, and so the compared object and the floating image appear to be the same. In Fig. 6 (b), we shifted the object position by a distance of $100 \mathrm{~mm}$ (the object distance is $100 \mathrm{~mm}$ ) from the first position toward lens1, so that the floating image looks bigger than the compared object. In Fig. 6 (c), we shifted the object position by a distance of $100 \mathrm{~mm}$ (the object distance is $300 \mathrm{~mm}$ ) from the first position away from the first lens, so that the floating image looks smaller than the compared object. In Fig. 6, the relative size depth cue [4] can be perceived, because the biggest image is nearer to the observer and the smallest image is farther from the observer.

Also, when the two lenses produce a floating image

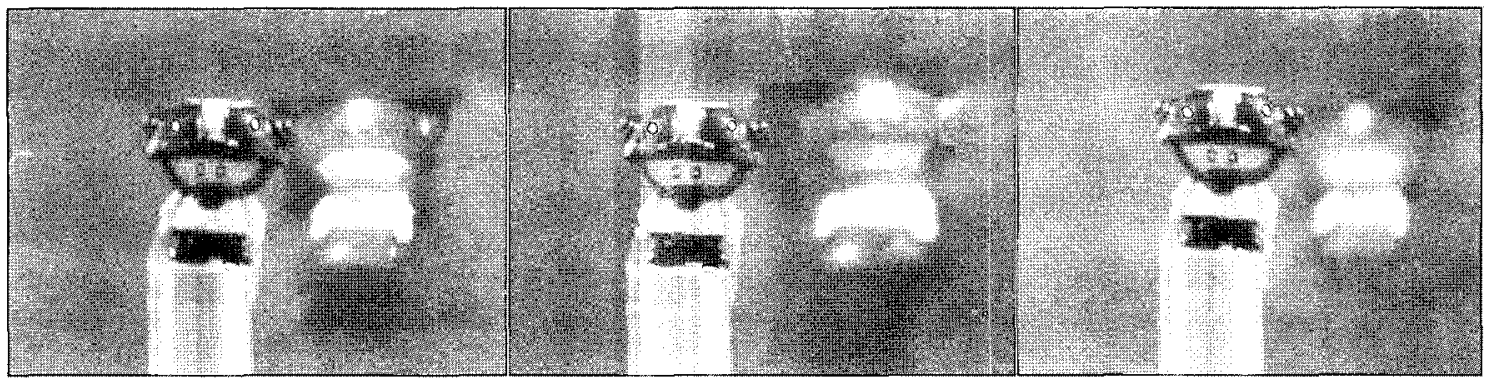

(a)

(b)

(c)

FIG. 6. Experiment result. (a) The object distance is $200 \mathrm{~mm}$. (b) The object distance is $100 \mathrm{~mm}$. (c) The object distance is $300 \mathrm{~mm}$.

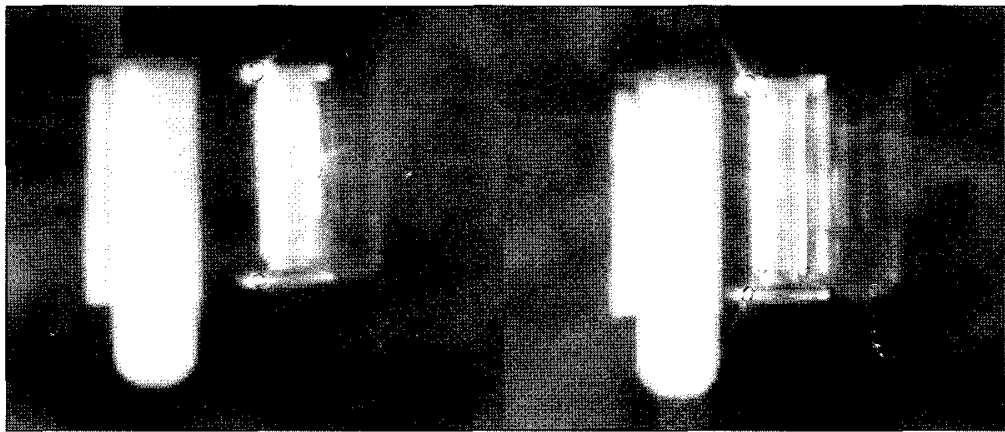

(a) (b)

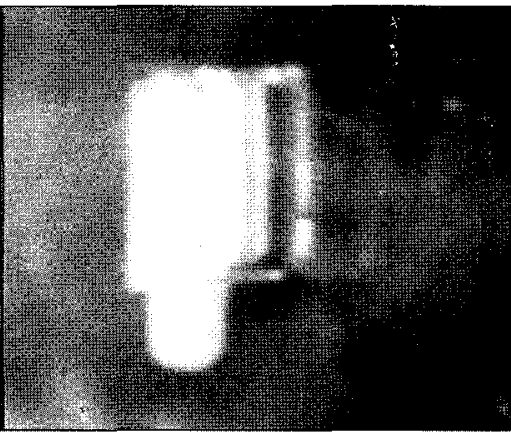

(c)

FIG. 7. Motion parallax depth. (a) Left view $10^{\circ}$ (b) Center view. (c) Right view $10^{\circ}$

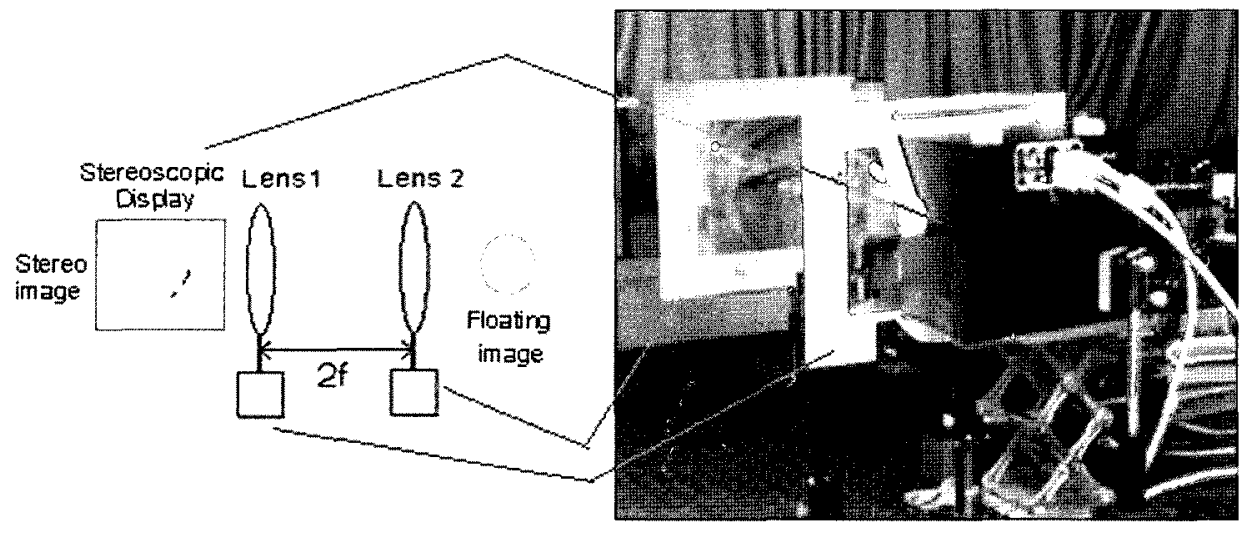

(a)

(b)

FIG. 8. Experimental system. (a) Schema of the proposed system (b) Experimental setup. 


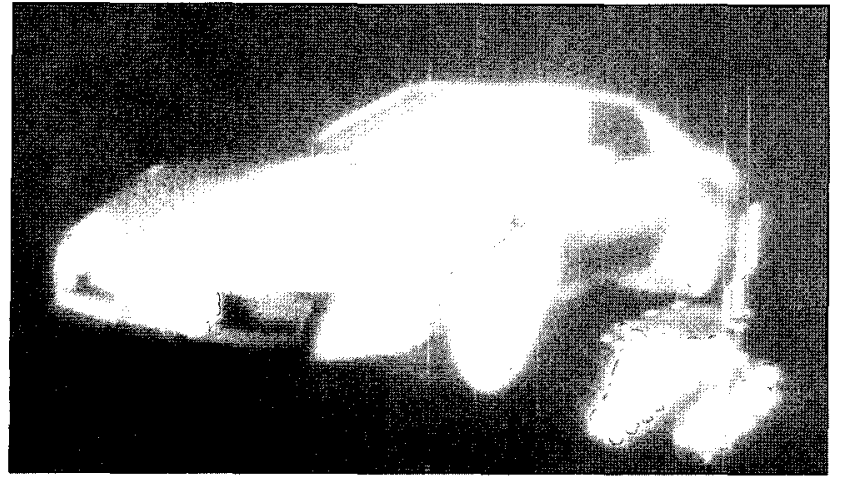

FIG. 9. Stereoscopic floating image.

from a real object, we can perceive the motion parallax depth $[4,7]$, because the two lenses can change the image position. The motion parallax is a very important depth cue. Another experimental result is shown in Fig. 7.

In step 2, we combined the two-lens system with a stereoscopic display. Figures 8 (a) and (b) show a schema of the proposed system and the experimental setup, respectively.

Once the stereoscopic display successfully produces a 3D image, the two lenses project it from inside of the stereoscopic display to mid air near the observer. When the viewer looks into the system with glasses, he or she can perceive the 3D image that is located in mid air. Therefore, the proposed system can produce stereoscopic floating images. An example of a stereoscopic floating image is showed in Fig. 9.

\section{CONCLUSION}

The two lenses eliminated all of the defects of the convex lens and concave mirror, because the distance magnification and height magnification of the two lenses is constant and the image distance is linearly related to the object distance or 3D image distance.

Since we can change the distance between the first lens and the stereoscopic display screen, the stereoscopic floating image distance is able to be controlled. The floating image distance is not changeable when using a large convex lens and concave mirror, because the height and distance magnification depend on the image distance.

When the viewer wears the polarized glasses and looks at the stereoscopic floating image, he or she can perceive the depth cues and the floating image.

\section{ACKNOWLEDGMENTS}

This work was supported by the Information Technology Research Center (ITRC) Support Program of the Ministry of Information and Communications in Korea, and by the Regional Research Centers Program of the Ministry of Education \& Human Resources Development in Korea.

*Corresponding author : ganbat@osp.chungbuk.ac.kr

\section{REFERENCES}

11) S.W. Min, M. Hahn, J. Kim, and B. Lee, "Three-dimensional electro-floating display system using an integral imaging method," Optics Express, vol. 13, no. 12, pp. 4358-4369, 2005.

[2] M. Halle, "Autostereoscopic displays and computer graphics," Computer Graphic, ACM Siggraph, vol. 31, no. 2, pp. 58-62, 1997.

[3] J.D. Lewis and C.M. Verber, A "True Three-Dimensional Display," IEEE Transactions on Electron Devices, vol. ED-18, no. 9, pp. 724-732, 1971.

[4] L. Lipton, Stereographics, Developers' Handbook (Stereographics Corporation), 1997.

[5] R. Ostnes, V. Abbott, and S. Lavender, "Visualisation techniques: an overview - part 2," The Hydrographic Journal, no. 114, pp. 4-9, 2004.

[6] Okoshi, T "Three Dimensional Displays," Proc. IEEE, vol. 68, pp. 548-564, 1980.

[7] Runar Ostnes, "Victor Abbott and Samantha Lavender," VISUALISATION TECHNIQUES: AN OVERVIEW PART 1," The Hydrographic Journal, no. 113, pp. 3-9, 2004.

[8] DANIEL G. ALIAGA, "Virtual and Real Object Collisions in a Merged Environment," Virtual Reality Software and Technology 94, pp. 287-298, 1994.

[9] Lenny Lipton, "Selection devices for field-sequential stereoscopic display: a brief history," in Stereoscopic Displays and Applications II, J O Merritt, S S Fisher, Proc. SPIE, vol. 1457, pp. 274-282, 1991.

[10] D.F. McAllister, "3D Displays," Wiley Encyclopedia on Imaging, pp. 1327-1344, 2002.

[11] Jihyun Kwak, Vs.V. Komar and Kyung-Tae Kim, "Synthesizing Intermediate Images Using Stereoscopic Images," J. Opt. Soc. Korea, vol. 6, pp. 143-149, 2002.

[12] Ho-In Jeon, Nak-Hee Jung, Jin-San Choi and Yoo-Seek, "Super Multi-View 3-D Display System Using Vibrating Scanner Array," J. Opt. Soc. Korea, vol. 4, pp. 37-42, 2000 . 